\title{
Automating the IRC Analysis within Eyringpy
}

\author{
Alan Quintal ${ }^{1}$, Eugenia Dzib ${ }^{1}$, Filiberto Ortíz $^{2}$, Pablo Jaque ${ }^{3}$, Albeiro Restrepo Cossio ${ }^{4}$, \\ and Gabriel Merino ${ }^{1}$ \\ ${ }^{1}$ Centro de Investigacion y de Estudios Avanzados del Instituto Politecnico Nacional \\ ${ }^{2}$ Universidad Juarez Autonoma de Tabasco \\ ${ }^{3}$ Universidad de Chile \\ ${ }^{4}$ Universidad de Antioquia, Colombia
}

March 17, 2021

\begin{abstract}
To analyze the evolution of a chemical property along the reaction path, we have to extract all the necessary information from a set of electronic structure computations. However, this process is time-consuming and prone to human error. Here we introduce IRC-Analysis, a new extension in Eyringpy, to monitor the evolution of chemical properties along the intrinsic reaction coordinate, including complete reaction force analysis. IRC-Analysis collects the entire data set for each point on the reaction coordinate, eliminating human error in data capture and allowing the study of several chemical reactions in seconds, regardless of the complexity of the systems. Eyringpy has a simple input format, and no programming skills are required. A tracer has been included to visualize the evolution of a given chemical property along the reaction coordinate. Several properties can be analyzed at the same time. This version can analysis the evolution of bond distances and angles, Wiberg bond indices, natural charges, dipole moments, and orbital energies (and related properties).
\end{abstract}

\section{Hosted file}

Main_Text.pdf available at https://authorea.com/users/402050/articles/513963-automating-theirc-analysis-within-eyringpy 